\title{
Broad-Band Diode-Pumped Ytterbium-Doped Fiber Amplifier with 34-dBm Output Power
}

\author{
J. M. Sousa, J. Nilsson, C. C. Renaud, J. A. Alvarez-Chavez, A. B. Grudinin, and J. D. Minelly
}

\begin{abstract}
We investigate a high-power diode-pumped doubleclad ytterbium-doped fiber amplifier with 34-dBm average output power and 1050-1095-nm bandwidth. A multidiode concentrator pumps the amplifier at $980 \mathrm{~nm}$, with $\sim 6 \mathrm{~W}$ of power launched into the inner cladding. Besides $\mathrm{CW}$-signals, we amplify pulses from a mode-locked laser to $1 \mathrm{~kW}$ of peak power with only minor nonlinear distortions as well as pulses from a $Q$-switched laser to $50 \mu \mathrm{J}$ of energy. Reflections and backscatter limit the gain of the amplifier to $40 \mathrm{~dB}$ for a pump power of $2.5 \mathrm{~W}$. For higher pumppowers than this, the amplifier started to self- $Q$-switch. The results are important for the development of cladding-pumped high-power fiber amplifiers.
\end{abstract}

Index Terms - Optical fiber amplifiers, optical fiber lasers, optical propagation in nonlinear media, optical pulse amplifiers, $Q$-switched lasers, ytterbium.

$\mathbf{H}$ IGH brightness, high power, if necessary tunable, nearinfrared sources find applications in many areas such as spectroscopy, laser and amplifier pumping, frequency conversion and medicine. For such sources, diode-pumped doubleclad fiber technology offers a unique combination of high efficiency, compactness, simple service-free operation, and high reliability. In the wavelength region 1-1.1 $\mu \mathrm{m}$, ytterbiumdoped fiber lasers and amplifiers are emerging as the best choice and have consequently, seen a rapid commercial development. ${ }^{1}$ However, experimental data on cladding-pumped $\mathrm{Yb}$-doped fiber amplifiers are sparse in the literature and at relatively low powers, e.g., $1 \mathrm{~W}$ [1].

In this letter, we present experimental data and discuss important features of a broad band high-power diode-pumped double-clad Yb-doped fiber amplifier (YDFA) with $34 \mathrm{dBm}$ $(2.8 \mathrm{~W})$ of saturated output power. We investigate its bandwidth and output power as a booster for a mode-locked Yb-doped fiber laser as well as for a tunable $Q$-switched Nd-doped fiber laser. We found that amplified spontaneous emission (ASE), self-induced $Q$-switching, and incomplete

Manuscript received August 17, 1998; revised September 29, 1998. The work of J. M. Sousa was supported by PRAXIS-XXI. This work was supported in part by EPSRC under the HIRAFS Project and in part by the Department of Trade and Industry.

J. M. Sousa is with INESC-UTOE-Grupo de Optoelectrónica, 4150 Porto, Portugal.

J. Nilsson is with the Optoelectronics Research Centre, University of Southampton, Southampton SO17 1BJ, U.K.

C. C. Renaud, J. A. Alvarez-Chavez, and A. B. Grudinin are with the Optoelectronics Research Centre, University of Southampton, Southampton SO17 1BJ, U.K.

J. D. Minelly is with Corning Inc., USA.

Publisher Item Identifier S 1041-1135(99)00347-X.

${ }^{1}$ Product catalogs of Polaroid, Inc., and IRE-Polus Group, 1998.

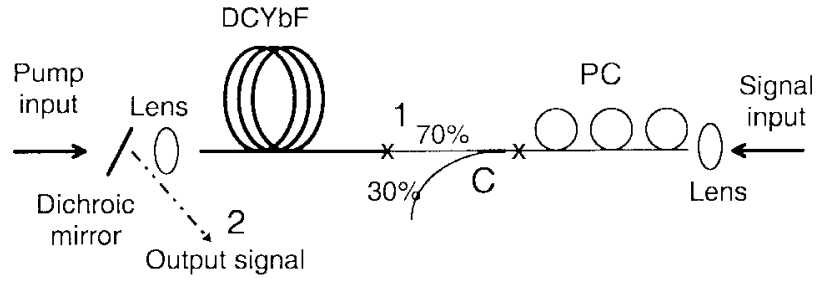

Fig. 1. Experimental setup. DCYbF: Double-clad Yb-doped fiber. C: 70/30 single-mode coupler. PC: Fiber polarization controller. $X$ : Splice points. Points 1 and 2 define the locations between which we measure the gain.

pump absorption are factors that limit performance of the amplifier in different modes of operation.

Fig. 1 illustrates our amplifier configuration. A laser diode concentrator from Diomed, Ltd., developed within the High Radiance Fiber Source (HIRAFS) project, with a bandwidth of $5 \mathrm{~nm}$ and a center wavelength of $979 \mathrm{~nm}$ was used as pump source. Approximately $6 \mathrm{~W}$ of the pump power was launched into the inner cladding of a double-clad fiber via a dichroic mirror. The in-house fiber had a silica inner cladding of 220 $\mu \mathrm{m}$ diameter and a silicone rubber external cladding for an NA of 0.4 for the pump. The aluminosilicate core was placed $30 \%$ off-center and had a diameter of $7.6 \mu \mathrm{m}$, an NA of 0.11 , and a cutoff wavelength of $1.0 \mu \mathrm{m}$. The $\mathrm{Yb}^{3+}$-concentration was $0.9 \%$ by weight. The fiber length was $14 \mathrm{~m}$ for $90 \%$ (10 $\mathrm{dB}$ ) of unbleached pump absorption at $976 \mathrm{~nm}$. However, our 5-nm-wide pump was centered at $979 \mathrm{~nm}$ where the absorption is lower. Thus, the fiber absorbed only about $70 \%$ of the pump (representative absorption and emission spectra can be found in [2]). A longer fiber could prove more efficient, but with a simultaneous, for us undesirable, shift of the gain spectrum to longer wavelengths [2].

The signal was launched into the Yb-doped fiber via a single-mode $70 / 30$ fiber coupler, with the $30 \%$ port used for monitoring the input signal power. The coupler was spliced to the double-clad fiber with an estimated splice loss of less than 1 dB. Points 1 and 2 in Fig. 1 constituted the amplifier's input and output ports for evaluating gain, input, and output powers. All fiber ends were angle cleaved to suppress reflections.

We first consider the bandwidth and output power of the amplifier. The signal source was a mode locked diode-pumped $\mathrm{Yb}$-doped fiber laser [3] which generated pulses of 10-20-ps duration [full-width at half-maximum (FWHM)] at an $80-\mathrm{MHz}$ repetition rate. The laser was tunable from 1020 to $1065 \mathrm{~nm}$. A bulk isolator with 5-dB insertion loss separated the signal source and the amplifier. Because the pulse rate is much faster than characteristic time-scales of the pumped Yb-system, the 


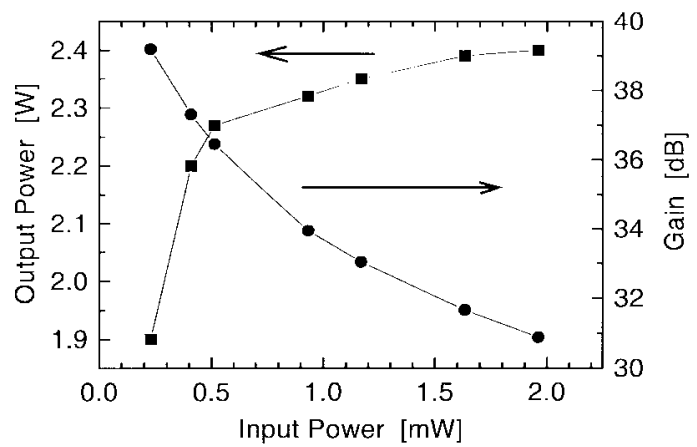

Fig. 2. Output power (squares) and gain (circles) versus input power at 1064 $\mathrm{nm}$.

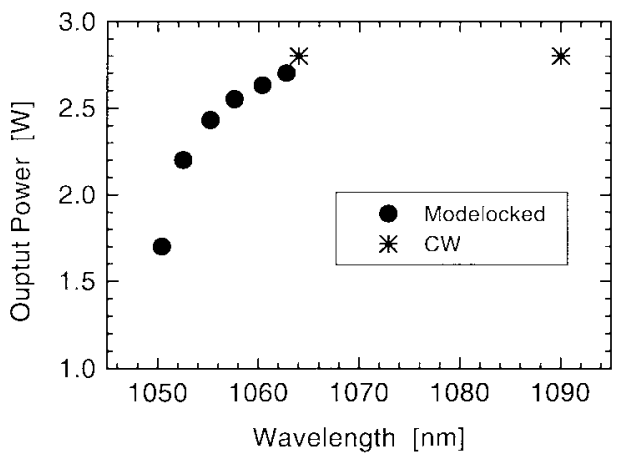

Fig. 3. Output power versus wavelength with a mode-locked (dots) and CW (asterisks) source.

pulse train is, in terms of average power, equivalent to a $\mathrm{CW}$ signal with the same power. The output power characteristics of the amplifier are shown in Figs. 2 and 3. The figures show a low-power gain of almost $40 \mathrm{~dB}$ and an output power of 2.4 $\mathrm{W}$, obtainable for input powers of $\sim 2 \mathrm{~mW}$ at $1064 \mathrm{~nm}$. This output power is achievable also at other wavelengths within the amplifier bandwidth; however, this would require launching a higher power than was available from the mode-locked laser.

Nonlinear pulse distortion due to self-phase modulation (SPM) is a main concern for short-pulse amplification. Fig. 4 shows input and output spectra of mode locked pulses. The output peak power is about $1 \mathrm{~kW}$. While the input spectrum corresponds to essentially transform-limited pulses, the output spectrum is somewhat broadened because of SPM. From the spectra, we estimate a nonlinear phase-shift of $0.7 \pi$ [4], which corresponds to propagation of pulses with this peak output power over an effective nonlinear length $L_{\text {eff }}$ of $1 \mathrm{~m}$ in our fiber. Assuming that the gain is homogeneously distributed along the fiber, we can also evaluate $L_{\text {eff }}$ as the inverse of the gain per unit length (in nepers per meter) for optical Kerr effect nonlinearities like SPM [4]. Thus, $L_{\text {eff }}=4.343 \times L / G=$ $4.343 \times 14 \mathrm{~m} / 40=1.5 \mathrm{~m}$, so the two ways of evaluating $L_{\mathrm{eff}}$ are in fair agreement.

The tuning range of the mode-locked laser did not cover the whole amplifier bandwidth. To explore the upper end of the amplifier's wavelength range, we instead used a diodepumped double-clad Nd-doped fiber laser, tunable by means of an external grating. The maximum output power was 2.8 $\mathrm{W}$ (asterisks in Fig. 3), higher than before because of a better

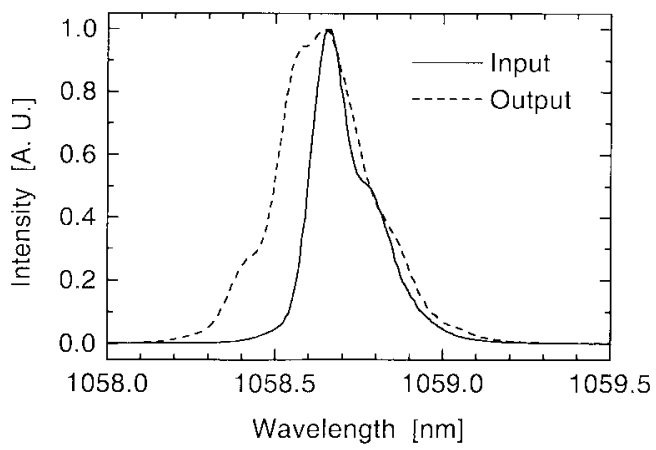

Fig. 4. Spectra of mode-locked pulses before and after amplification.

pump alignment. The launched signal power was large enough to saturate the amplifier even near the edge of the amplifier's bandwidth. Again, this higher output power is available for all wavelengths within the amplifier bandwidth for high enough signal input powers.

We next discuss amplification of $Q$-switched pulses. The signal source was a cladding pumped $Q$-switched Nd-doped fiber laser [5], generating 130-ns pulses (FWHM) with a 30$\mathrm{kHz}$ repetition rate at $1064 \mathrm{~nm}$. We launched $10 \mathrm{~mW}$ of average power, some $20 \%$ of which was in the form of ASE between pulses. The repetition rate was low enough to allow for almost complete gain recovery between pulses, but the gain was still compressed to $28 \mathrm{~dB}$ by the ASE. The output power was $2.8 \mathrm{~W}$, divided nearly equally between the 130-ns pulses and ASE. Thus, the output pulse energy was approximately $50 \mu \mathrm{J}$, which is close to the highest output energy extracted from YDFA's [6], and now at a much higher repetition rate. We note that the intrinsic saturation energy $E_{I S}$ becomes 33 $\mu \mathrm{J}$ for our fiber at $1064 \mathrm{~nm}$. Given that the gain is related to the extractable energy $E_{\text {ext }}$ by $G=4.343 \times E_{\text {ext }} / E_{I S}$ [6], the unsaturated gain of $40 \mathrm{~dB}$ corresponds to an extractable energy of $300 \mu \mathrm{J}$ from the fiber. Under more favorable conditions, e.g., a source with higher-energy pulses and no ASE, this fiber has, therefore, a potential to amplify pulses to significantly higher energies than we obtained. In practice, however, we have found this to be difficult.

One of the most interesting features of the high-power YDFA was its behavior in the absence of any input signal. For pump powers below $2.5 \mathrm{~W}$, the amplifier produced, as expected, ASE with single-ended power of $250 \mathrm{~mW}$ and a 3$\mathrm{dB}$ bandwidth of $20 \mathrm{~nm}$. Fig. 5 illustrates an ASE spectrum, as measured at the signal input end. The ASE spectrum gives a good indication of the small-signal gain spectrum (typically, the spectral dependence of the spontaneous emission factor $n_{\mathrm{sp}}$ is small over moderate wavelength ranges).

For launched pump powers above $2.5 \mathrm{~W}$, however, the amplifier self- $Q$-switched, producing $\sim 2$ ns pulses with peak power of 4-5 kW. At maximum pump power, the two-ended average output power was $2 \mathrm{~W}$. The 2-ns-long (FWHM) pulses had a $8-\mathrm{kHz}$ pulse repetition rate, although the pulse-to-pulse time interval fluctuated by up to $5 \%$. This behavior indicates that the pulsing arises from distributed back-scattering [5], [7]. In an amplifier context, the pulsation limits the smallsignal gain (to $40 \mathrm{~dB}$ ) that can be reached in the amplifier. 


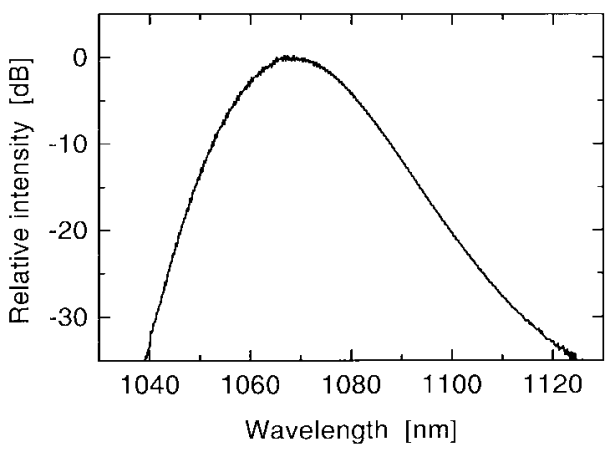

Fig. 5. Single-pass ASE spectra from the signal input end of the YDFA at 2.5-W launched pump power.

For high-energy applications, it also limits the energy that can be stored in the device, since a higher gain implies a higher stored energy. Furthermore, for high average powers, a higher small-signal gain would mean that correspondingly lower input powers would suffice to reach saturation. The amplification bandwidth would also increase.

Besides the distributed back-scattering, also fiber facet reflections and reflections off lenses can give rise to feedback. While the angle-cleaves at the fiber ends were sufficient to suppress any reflections there, we still suffered from residual reflections from lenses. Such reflections can limit the gain by enhancing the ASE and reducing the laser threshold. Isolators can eliminate back-reflections, but high-performing pig-tailed isolators are not readily available at the powerlevels and wavelengths of our amplifier, and they are anyhow incompatible with end-pumping of double-clad fibers. We note that $47 \mathrm{~dB}$ of small-signal gain was recently reported from a diode-pumped double-clad ytterbium-doped fiber without any isolators [8]. It is unclear to us why our amplifier failed to reach such high gain.

In summary, we have investigated a diode-pumped highpower broad-band double-clad Yb-doped fiber amplifier with 34- $\mathrm{dBm}$ saturation output power and 45-nm bandwidth, from
1050-1095 nm. We also demonstrated amplification of $Q$ switched input signal pulses to $50 \mu \mathrm{J}$ of energy. Without any input signal, the amplifier started to self-pulsate, with feedback probably arising from distributed back-scattering. Although this shows the ability of the amplifier to extract high-peak power, high-energy pulses, it also ultimately limits the smallsignal gain and bandwidth of the amplifier. Since even small amounts of back-scattering and reflections at fiber ends or optics can enhance this pulse regime care must be paid to prevent them.

\section{ACKNOWLEDGMENT}

The authors wish to thank Z. J. Chen for valuable discussions and for assistance with the $\mathrm{Nd}$-doped fiber laser. J. A. Alvarez-Chavez is grateful for the support of CONACYT and CICESE, both of Mexico. The ORC is an EPSRC-funded interdisciplinary research center.

\section{REFERENCES}

[1] S. V. Chernikov, J. R. Taylor, N. S. Platonov, V. P. Gapontsev, P. J. Nacher, G. Tastevin, M. Leduc, and M. J. Barlow, "1083 nm ytterbium doped fiber amplifier for optical pumping of helium," Electron. Lett., vol. 33, pp. 787-789, 1997.

[2] R. Paschotta, J. Nilsson, A. C. Tropper, and D. C. Hanna, "Ytterbiumdoped fiber amplifiers," IEEE J. Quantum Electron., vol. 33, pp. 1049-1056, 1997.

[3] J. Porta, A. B. Grudinin, Z. J. Chen, J. D. Minelly, and N. J. Traynor, "Environmentally stable picosecond ytterbium fiber laser with a broad tuning range," Opt. Lett., vol. 23, pp. 615-617, 1998.

[4] G. P. Agrawal, Nonlinear Fiber Optics. San Diego, CA: Academic, 1995.

[5] Z. J. Chen, A. B. Grudinin, J. Porta, and J. D. Minelly, "Enhanced Q switching in double-clad fiber lasers," Opt. Lett., vol. 23, pp. 454-456, 1998

[6] J. Nilsson, R. Paschotta, J. E. Caplen, and D. C. Hanna, "Yb3+-ringdoped fiber for high-energy pulse amplification," Opt. Lett., vol. 22, pp. 1092-1094, 1997.

[7] S. V. Chernikov, Y. Zhu, J. R. Taylor, and V. P. Gapontsev, "Supercontinuum self- $Q$-switched ytterbium fiber laser," Opt. Lett., vol. 22, pp. 298-300, 1997.

[8] L. Goldberg, J. P. Koplow, and R. P. Moeller, "High-power superfluorescent source with a side-pumped Yb-doped double-cladding fiber," Opt. Lett., vol. 23, pp. 1037-1039, 1998. 